\title{
Clustering Algorithm for Energy- Efficient Wireless Sensor Network
}

\author{
${ }^{1}$ Asha Rawat, ${ }^{2}$ Dr. Mukesh Kalla \\ ${ }^{1} \mathrm{Ph}$. D. Scholar, Department of Computer Engineering, Sir Padampat Singhania University, Udaipur, Rajasthan, \\ India. \\ ${ }^{2}$ Associate Professor, Department of Computer Engineering, Sir Padampat Singhania University, Udaipur, \\ Rajasthan, India.
}

Article History: Received: 11 January 2021; Accepted: 27 February 2021; Published online: 5 April 2021

\begin{abstract}
Wireless networks data aggregation allows in-network processing, reduces packet transmission and data redundancy, and thus helps extend wireless sensor systems to the full duration of their lives. There have been many ways of dividing the network into clusters, collecting information from nodes and adding it to the base station, to extend wireless sensor network life. Certain cluster algorithms consider the residual energy of the nodes when selecting clusterheads and others regularly rotate the selection head of the cluster. However, we seldom investigate the network density or local distance. In this report we present an energy-efficient clustering algorithm that selects the best cluster heads of the system after dividing the network into clusters. The cluster head selection depends on the distance between the base station nodes and the remaining power of this approach.Each node's residual energy is compared to the node count. Our results show that the solution proposed more efficiently extends the life of the wireless sensor network. The algorithm prolongs the life and effectiveness of the Wireless Sensor Network.
\end{abstract}

Keywords: Data Aggregation, Energy Efficiency, Residual Energy, Clustering, Wireless Sensor Networks.

\section{Introduction}

Wireless sensor networks (WSNs) have begun to be tremendously research-driven in different applications. Scientists have consistently concentrated on the energy requirement of sensor networks. Data aggregation is a successful energy-saving strategy for improving sensor network life. Among different methodologies, Hierarchical cluster-based data aggregation protocols are generally utilised because of high dependability and adaptability. However, in the cluster-based sensors network there is a problem of unbalanced energy dissipation [14].

Countless sensor nodes driven by the battery are transmitted to a wireless sensor system and the energy saving of the nodes becomes a vital problem. Various routing techniques to reduce energy use were proposed. Taking this fact into account, if it is very clear that a sensor node needs to use energy before it transmits the nodes, it will help to gain an insight into the efficient energy topology of the network[15].

Energy harvesting $(\mathrm{EH})$ is growing as a promising answer to understanding the energy-efficient green communication in the Wireless Sensor Networks (WSNs). Non-EH nodes can save energy by using EH nodes as relay nodes and it is necessary to relay the valuable energy with better performance. Many times, the rate of EH charge is low because the current charging strategies cannot be well modified over environmental energy, therefore higher charging levels are essential[13].

Intelligent transportation frameworks were generally shipped to wireless sensor networks (WSNs). The WSN (BF-WSN) battery-free scavenge climate energy. The conveyance of data with low energy usage is important for BF-WSN nodes which are capable of collecting radio frequency (RF) energy. Some existing baseband coding or tweaks for carriers show the energy use difference (ECD) between bit 0 and bit 1 . One model is the base-band encoding of the FM0, usually applied in radio frequency backscatters to recognizable proof frameworks and wireless ID detection. The sending of bit 0 in FM0 devours much more remarkable energy than bit 1 [16].

Sensor nodes closer to the Basic Station (BS) deplete their energy quicker than far away nodes during multi-hop clustering. The primary cause of this uneven energy consumption is transmitting own and other node data via the nearer nodes. So the nodes closer to the BS die fast and disconnect the network, although most nodes have sufficient energy for communication. The problem of this type is known as the WSN hot spot [17] problem.

Given that providing the collected data in the sink is one of the WSN's major tasks, optimizing network communication in order to improve energyefficiency is an important concern of the routing protocol. In this paper, the Chord protocol is used to develop a new protocol for data delivery in WSNs. In contrast to the majority of existing DHT-based routing systems in the literature, our proposal brings a superlaying structure closer to the base network where overlay nodes are interconnected, given their physical proximity. In particular, it could minimize the energy consumed by communication, loading and transmission delay [18]. This would guarantee a number of benefits.

Energy savings are the prime job in WSN, and the selection of the cluster head $(\mathrm{CH})$ is a key factor here. Different parameters, such as node energy, Euclidean distance and randomness, are used for the $\mathrm{CH}$ selection. In 
this paper we propose the LEACH (RCH-LEACH) algorithm for residual energy-based cluster management to stabilize the formation of a network cluster. This algorithm employed few additional parameters for the selection of CHs, for example threshold energy, node residual energy, and optimal cluster number [19].

\section{Literature Review}

We propose in this document an energy efficient multi-hop transmission strategy with the cluster phase of the organization. Our main goal is to develop a new transmission technology that minimizes power consumption. It is a multi-hop transmission technique where the selected transmission relays offer the lowest transmission power while maintaining the SNR level equal to the $\mathrm{S}^{\text {th }}$ threshold. Therefore, in comparison to the direct transmission and transmission the proposed transmission technique can significantly reduce energy consumption when the $\mathrm{CH}$ node is selected randomly inside the cluster. Simulation results show that a significant increase in energy saving is the addition of an organization cluster phase before data transmission.

This study [1] broadly examines and speaks of the algorithms that have been developed to deal with the problems and systems that have inspired time synchronization over WSN and the transmission condition properties of deafness and packet collider by integrating energy-efficient fires. In particular, it is an exhaustive examination incorporating the transmission state instrument, points of interest and detrimental effects of past related work. The report helps scientists to (1) avoid the uncertainty that occur in the transmission state of WSNs, (2) to prevent the packet collision during the transmission in WSNs, and (3). It also contains the recommendation for future research of several relevant, open-ended issues.

In this paper [2] we propose a multi-hop transmission technology based on the Dijkstra algorithm for the Wireless Sensor Networks (WSN). A WSN of N sensor nodes is considered. Firstly, we group the sensor nodes to clusters based on their position in the area of monitoring, then arrange the nodes for each cluster, choose the appropriate node as a cluster head and classify the remaining nodes into active nodes and sleeping nodes. We then choose the set of reliable relays to transmit data with the least transmission power. Our proposed algorithm for relay selection is based on Dijkstra. The paper is mainly aiming at defining a new transmission strategy which improves the results of our previous work[2] by reducing power consumption more effectively.

This paper[3] examines WSNs' energy-efficient cooperative communication across frequency-selective fading channels. For systems over frequency-selective fading channels, the closed-form bit error rate expressions are derived. The optimum transmission power allocation solutions and the selection of the Partner Node will be provided to explore fully the energy conservation potential of cooperative communications. Moreover, it is proved that chip-interleaving techniques in WSN's with flat fading channels can be used to significantly improve communication quality. Thus this paper examines the energy saving potential in frequency selective decomposition of chip-interleaved transceivers in WSN.

This article [4] proposes a new energy-efficient routing technology scheme for the remote monitoring of agriculture in WSN. In this system, a crop field has various sensor node types with limited source of power, randomly positioned for sensing different soil characteristics like salinity, humidity, temperature and $\mathrm{PH}$. In addition to grid-shaped clusters, this system includes a tolerance of sensory data of the above-mentioned parameters to offer efficient clustering and percentage tolerance. The efficiency of the proposed system was assessed by the number of live nodes and the number of data packets transmitted and received.

In the case of MECA and PEGASIS,[5] we propose an energy-efficient lifetime improvement Strategy for WSNs. The objective of this algorithm is to minimise and balance the use of energy for all sensor nodes and cluster heads. We split the network into a series of equal groups and multi-hop routing is used to save energy in the intra-cluster routing. A chain such as PEGASIS is connected to the cluster heads so that a distant cluster head can not be sent to the sink node. Results of simulation show that the routing algorithm proposed is less consumed than traditional routing algorithms such as LEACH.

This Paper [6] aims mainly at finding ways of improving WSNs and increasing their lives. In this paper, we propose a compressive sensing clustering algorithm, using certain clustering algorithms to significantly save WSN energy. Furthermore, in various sensing conditions we show the optimal number of clusters and optimize energy consumption for WSNs.

The papers proposed[7] that the energy consumption should be deduced and that the lifetime of the Wireless Sensor Network (WSN) connection set be improved. The data is transferred throughout the system via each sensor to a single process center that uses all communicated data to view or find the occasion for individual settings. To reduce the sensors' restricted energy capability, the message travel method should be premeditated. Cluster sensors were strongly grouped into teams so that sensors would only be available with contact information to cluster heads so that the cluster heads could exchange information to the process center, saving energy. The primary aim of this paper is to begin an examination in the literature of Wireless Sensor Networks of cluster Algorithms and cluster parameters. Additionally, WSNs propose energy-efficient cluster algorithms.

This paper[8] produces an overall energy efficient mathematical optimisation model in hybrid SDWRSNs that provides a sketch and paradigm for hybrid SDWRSN energy savings issues. We subsequently presented the hybrid SDWRSN energy-saving traffic planning (ETS). The ETS algorithm takes account of the losses of 
charging and new requirements of energy efficiency and calculates the flows from both (direct) and (indirect) SDN nodes. The results of the simulation show that the energy efficiency of hybrid SDWRSNs can be significantly improved by up to $20-40 \%$ on traditional routes. For this reason, ETS can make the network work much longer-term more consistently and efficiently.

This paper proposes[9] a new energy efficient routing system in WSN for remote monitoring of agriculture. In this system, a crop field has various sensor node types with limited source of power, randomly positioned for sensing different soil characteristics like salinity, humidity, temperature and PH. In addition to grid-shaped clusters, this system includes a tolerance of sensory data of the above-mentioned parameters to offer efficient clustering and percentage tolerance.

Leach is a protocol for the hierarchy of less energy adaptive clustering. The primary objective of cluster-based sensor networks is to reduce system delay and energy consumption. Leach is an energy- efficient and scalable routing and fair media accès cluster-based protocol for micrometric sensor networks. In the network of wireless sensors, many improvements are made. In the network of wireless sensors, safety is very important.

The main issue in this paper[11] is energy efficiency in developing WSN (Wireless Sensor Network) clusterbased routing protocols, since sensor nodes have low power levels. During data diffusion and receipt, sensor nodes mainly consume energy. The Wireless Sensor Network routing protocols should be energy-efficient, so that the lifetime of the individual node is not only increased, but that all wireless sensor networks can last longer. There are different routing protocols, but we selected LEACH because both the energy efficiency and the lifetime of the network are better performed.

A distributed, independent energy system (DAEMS) is introduced in this paper[12]. The DAEMS is intended to be autonomous, scalable and reliable in energy trading, customer usage optimization and DER management. The aim of this paper will therefore be to create a technical platform to facilitate energy trade, thereby creating an energy market and enabling consumers to become involved in the green economy. This type of technical platform can be achieved with the formulation of energy efficiency algorithms for customers and the finding of suitable DER management methods in terms of energy consumption, generation and connectivity in real time.

\section{ExistingSystem}

A multihop transmission strategy with an organizational cluster phase is proposed by the existing system. Two steps are taken to implement this communication strategy. The first phase attempts to select the $\mathrm{CH}$ node and classify the other nodes into sleeping and active nodes within the cluster. The second step consists in selecting the set of reliable relays that offer the least power to transmit while keeping the SNR equal to the $\alpha$ th threshold. This set of nodes is used to transmit to facilitate communication with the source destination. The system first developed the cluster organization phase, and then the algorithm for relay selection. Our proposed multi-hop transfer strategy based on SNR threshold criteria is comprehensive.

\section{A. Cluster Organization Phase}

We have carried out a process before data transmission that allows the cluster to organize the nodes. This process takes two steps: first, we choose a node in the cluster as the head of the cluster. With possible minimal energy this node can communicate with any other node. The main function of the chosen $\mathrm{CH}$ node is to transmit data from the source node to a maintenance center to take the appropriate response. We suggest two methods based on the distance criteria in order to choose the $\mathrm{CH}$ node. So first of all, we calculate the distance of the matrix between all the cluster nodes. The first method is to choose the node with the lowest average distances from all nodes as $\mathrm{CH}$. In the second method, the node with the maximum distance from all nodes is chosen as $\mathrm{CH}$. The second step is the classification of the nodes in the cluster organization.

\section{B. Relay selection algorithm}

Our proposed technology aims at maintaining at the receiver a minimum SNR equal to $\alpha$ th and minimizing the total power consumption. In order to transmit the detected data to the cluster head with minimum power, we select a set of reliable transmissions to help source node.

\section{Transmission Strategy}

A cluster organization phase precedes the proposed transmission strategy. This enables the $\mathrm{CH}$ node to be chosen and two sleeping and active nodes classified into two groups. As cooperation is not always more energized than direct transmission, we did not restrict ourselves to co-operative transmission in our transmission strategy, but used both systems of transmission. 


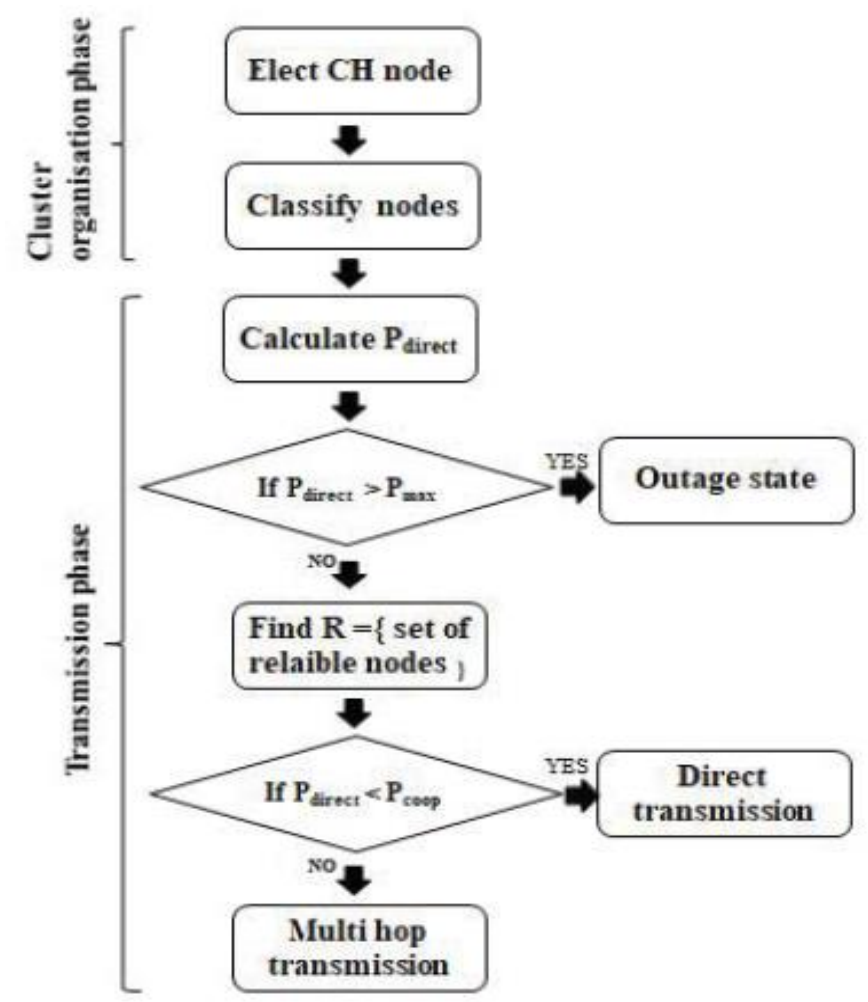

Fig. 1 Transmission Strategy Flow Chart

\section{Implementation Details}

The network and energy consumption model are defined below before detailing our proposed algorithm. respectively.

- $\quad$ The Base Station can be located anywhere in the area and after deployment the nodes are placed stationary.

- $\quad$ A 2D matrix consisting of the unique IDs in every node is deployed in the graph and the $\mathrm{Xi}$ and $\mathrm{Yi}$ values are mentioned in TABLE I.

- When initially deployed, all sensor nodes have variable energy levels, equivalent to one 1 Joule (J).

- $\quad$ The sensor nodes are assumed to be of the same communications range and indicated by R.

- A sub-set of the neighboring nodes denoted as $\mathrm{N}(\mathrm{v})$, which can be reached by $\mathrm{v}$ in $\mathrm{R}$ is each node $\mathrm{v}$.

- In each BS round, the IDs and the distances between nodes and their neighbours are sent for each node.

- $\quad$ Sensor nodes transmit their data in a single-hop way to their respective CHs.

$E_{T x}(l, d)=\left\{\begin{array}{cc}l E_{\text {elec }}+l \epsilon_{f s} d^{2}, & d<d_{o} \\ l E_{\text {elec }}+l \epsilon_{m p} d^{4}, & d \geq d_{o}\end{array}\right.$

$\mathrm{E}_{R x}(l)=l \mathrm{E}_{\text {elec } \ldots . . .(2)}$

$d_{o}=\sqrt{\frac{\epsilon_{\mathrm{fs}}}{\epsilon_{\mathrm{mp}}}}$

The LEACH algorithm is used to have the same energy model. The energy consumption per bit of the transmitter and receiver model is displayed as the energy used to transfer the l-bit packet over distance $d$ in Equation (1) and in Equation (2) with Eelec for receipt. The parameters $\epsilon_{\mathrm{fs}}$ and $\epsilon_{\mathrm{mp}}$ are the power amps in the free space (2 loss), and the multipath (4 loss) as per the distance from the equation (3).

Table 1. Node Representation 


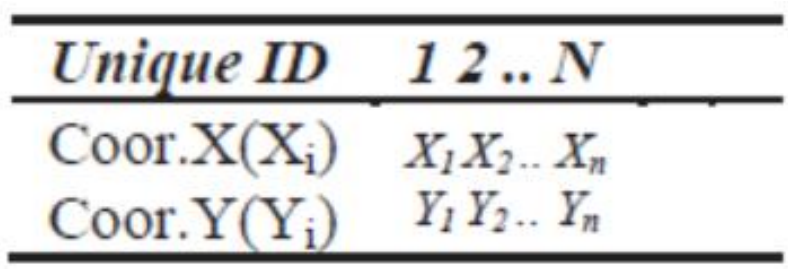

In this section, we introduce our new Improved Energy Efficient Clustering Algorithm. IEECA is a distributed clustering approach involving local decision making for the choice of $\mathrm{CHs}$ and residual energy consideration in nodes. At the beginning of each clustering round, the base station provides all sensing nodes with information on each neighbours. This means that every node knows both the position and the residual energy of each node within its range before CH's are elected. The two important steps are our main idea:

1. In view of the above parameters, each node will choose itself as the candidate and update its position. This means that each sensor is either a $\mathrm{CH}$ or a member at the end of this step.

2. Based on the distance between nodes and CHs clusters will be formed.

First, every sensor becomes a candidate and this status will be changed or not depending on the comparison. If I find a node that has greater energy than its own, it will change its status as a member, otherwise the density is compared, and if the sensor node has a higher density, the sensor node I will be regarded as more eligible to be $\mathrm{CH}$. Every node will send a broadcast message to inform other nodes about their status if all nodes configure their status.

The IEECA algorithm uses a probabilistic method for selecting CHs, comparing all of the sensor nodes and then we are sure that only one $\mathrm{CH}$ has been selected in the field $\mathrm{R}$. The number of $\mathrm{CHs}$ is based on the range chosen. If the range is reduced, this number will increase.

\section{Algorithm 1: Energy Efficient Clustering Algorithm}

After this algorithm of clustering and division of nodes into numbers of clusters is implemented, the cluster head is chosen on the basis of distance, the key distribution is carried out in each node via the base station, and the route is generated from each node to the base station. Encrypt data using the private key's Paillier Cryptosystem algorithm.

Send all the data to the base station afterwards. Send all the data to the base station afterwards. When the data cluster head is sent to the cache, the data is first sent from the cache and then the data is sent from $\mathrm{CH}$. The dataset with the appropriate keys decrypts the data.

1. Generate the network diagram as diagram $\mathrm{G}(\mathrm{v}, \mathrm{e}), \mathrm{V}$ is the vertical / node and $\mathrm{E}$ the edges.

2. Implement the clustering algorithm over the nodes and divide the nodes into a cluster number.

3. Select the Efficient Cluster Head based on energy, number of neighbours and distance from the base station.

4. Execute the key distribution through the base station at each node.

5. $\quad$ Execute from each node to the base station the route generations.

6. Create data in each node and encrypt the data with the base station's public key.

7. Calculate the encrypted data hash value and record the time stamp.

8. Send the individual data from every member of the cluster to the cluster head. Also save data in cache memory when the cluster head capacity exceeds the limit.

9. Collect all the information at the head of the cluster.

10. Send data to the base station and aggregate all data. The base station accepts the cluster head data.

\section{Experimental Setup}

The Experiment is conducted using JUNG Simulator on the Windows platform, the System constructs on Java framework (JDK version 8). The Netbeans tool (version 8.1) is used for development. System requires no specific hardware for the application to run any standard machine.

\section{Simulation Result}

Following are Results generated during the implementation of the system. 


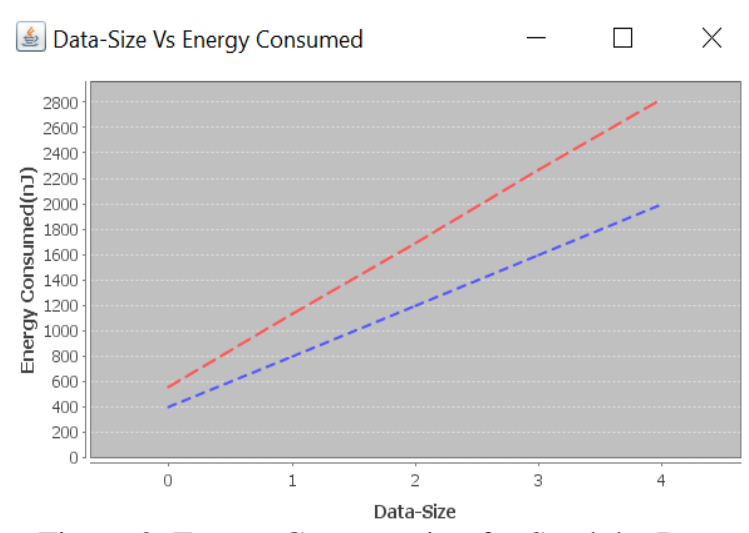

Figure 2: Energy Consumption for Send the Data

The outcomes appeared in Figure 2 and Figure 3 gives the Energy and Time Consumption while sending the information. For better investigation of the outcomes we have demonstrated the consequences of 5 tries. Presently the Energy and Time expended amid the transmission incorporates the assets used in delays. We characterize that handling delay signifies the execution time part nodes require to create their ciphertexts and comparing marks. The aggregation delay is estimated by deciding the time spent checking the marks from part nodes, amassing ciphertexts and marks, and creating the mark of the accumulated outcome. Unscrambling delay shows the time spent on in the end picking up the first information for the BS by confirming the totaled marks and decoding accumulated ciphertexts.

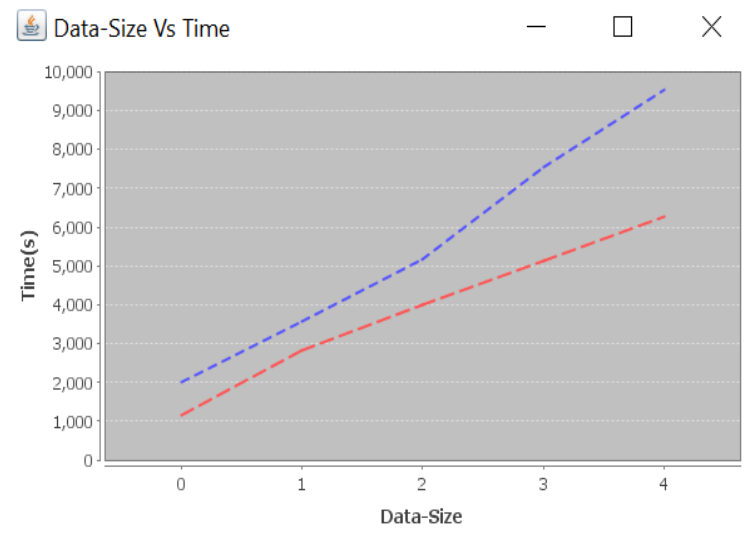

Figure 3: Time Consumption for Send the Data

To further evaluate the results, the system has been evaluated for different set of nodes for both the existing system and proposed system. The System has been tested for 30 nodes, 50 nodes, 80 nodes and 100 nodes. The results obtained represented in following figure 4 and 5.

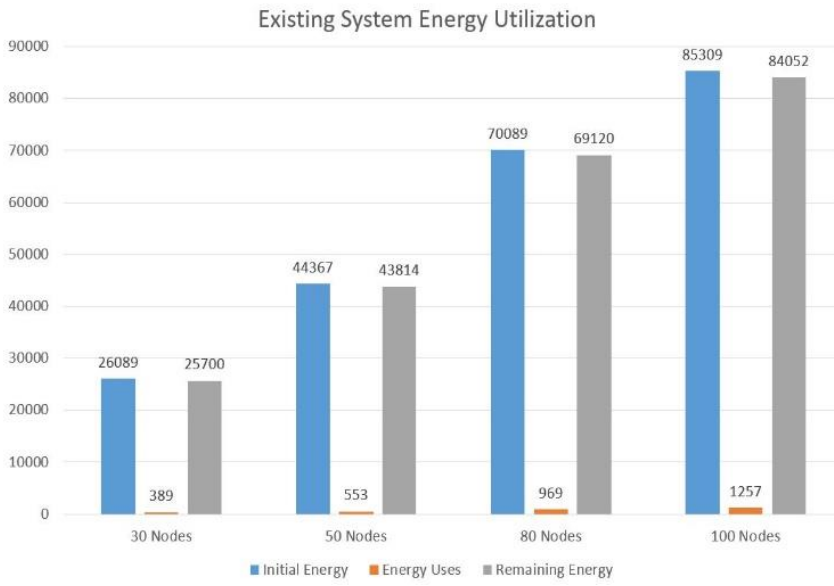

Figure 4. Energy utilization for Existing System 


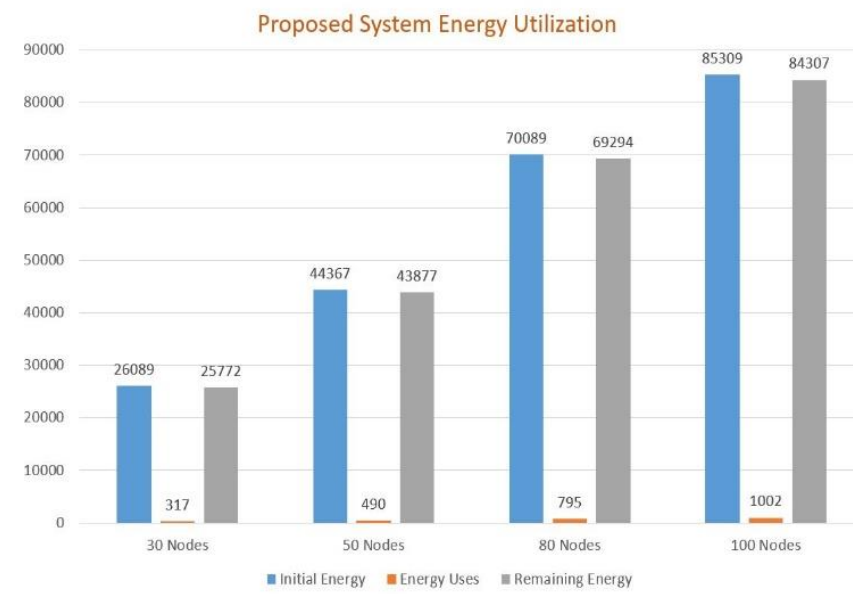

Figure 5. Energy utilization for Proposed System

\section{Conclusion}

Energy is one of the most critical resources of wireless sensor networks. Energy conservation was the main objective of literature for optimization of algorithms clustering. The algorithm's main point is to allow the whole sensor nodes to compete at random in the network. In accordance with the residual energy and local distance, we proposed the EECA Algorithm to select the cluster head for the $\mathrm{CH}$ to be used. The simulation results have shown that the EECA algorithm enhances network life. The security mechanism raises a key question for future work, given the operation of sensor nodes and the need to collect information to guarantee confidentiality, integrity, effective and secure routing in order to avoid malicious node attacks. The security mechanism is also important for future work.

\section{References}

1. Z. G. Al-Mekhlafi, Z. M. Hanapi and A. M. Shamsan Saleh, "Firefly-Inspired Time Synchronization Mechanism for Self-Organizing Energy-Efficient Wireless Sensor Networks: A Survey," in IEEE Access, vol. 7, pp. 115229-115248, 2019, doi: 10.1109/ACCESS.2019.2935220.

2. M. Abderrahim, H. Hakim, H. Boujemaa and F. Touati, "Energy-Efficient Transmission Technique based on Dijkstra Algorithm for decreasing energy consumption in WSNs," 2019 19th International Conference on Sciences and Techniques of Automatic Control and Computer Engineering (STA), Sousse, Tunisia, 2019, pp. 599-604, doi: 10.1109/STA.2019.8717210.

3. Tian, J., Berber, S. \& Rowe, G. Energy Efficient Cooperation with Chip-Interleaved Transceivers in WSNs Over Frequency-Selective Fading Channels. Wireless Pers Commun 95, 3933-3953 (2017). https://doi.org/10.1007/s11277-017-4033-6

4. U. K. Paul and S. Chattopadhyay, "An energy saving routing scheme for WSN based crop field monitoring system," 2016 IEEE Annual India Conference (INDICON), Bangalore, 2016, pp. 1-5, doi: 10.1109/INDICON.2016.7839097.

5. J. Wang, J. Cao, Y. Cao, B. Li and S. Lee, "An Improved Energy-Efficient Clustering Algorithm Based on MECA and PEGASIS for WSNs," 2015 Third International Conference on Advanced Cloud and Big Data, Yangzhou, 2015, pp. 262-266, doi: 10.1109/CBD.2015.49.

6. Nguyen M.T., Teague K.A. (2019) Optimizing Number of Clusters for Energy Saving Purpose in Wireless Sensor Networks. In: Fujita H., Nguyen D., Vu N., Banh T., Puta H. (eds) Advances in Engineering Research and Application. ICERA 2018. Lecture Notes in Networks and Systems, vol 63. Springer, Cham. https://doi.org/10.1007/978-3-030-04792-4_59

7. D. L. Reddy, V. Aran, A. V. Paramkusam and N. Nagaraju, "Wireless sensor networks algorithms to improve energy efficiency: Review," 2017 International Conference on Intelligent Sustainable Systems (ICISS), Palladam, 2017, pp. 1164-1166, doi: 10.1109/ISS1.2017.8389369.

8. X. Ma, Y. Wei and N. Yang, "Energy-saving traffic scheduling in hybrid software defined rechargeable WSNs," 2017 IEEE 28th Annual International Symposium on Personal, Indoor, and Mobile Radio Communications (PIMRC), Montreal, QC, 2017, pp. 1-6, doi: 10.1109/PIMRC.2017.8292658.

9. K.A. Shim, C.M. Park, A secure data aggregation scheme based on appropriate cryptographic primitives in heterogeneous wireless sensor networks, IEEE Parallel Distrib. Syst. 26 (8) (2015) 2128-2139.

10. S. K. Singh, P. Kumar and J. P. Singh, "A Survey on Successors of LEACH Protocol," in IEEE Access, vol. 5, pp. 4298-4328, 2017, doi: 10.1109/ACCESS.2017.2666082.

11. M. Aslam, N. Javaid, A. Rahim, U. Nazir, A. Bibi and Z. A. Khan, "Survey of Extended LEACH-Based Clustering Routing Protocols for Wireless Sensor Networks," 2012 IEEE 14th International Conference 
on High Performance Computing and Communication \& 2012 IEEE 9th International Conference on Embedded Software and Systems, Liverpool, 2012, pp. 1232-1238, doi: 10.1109/HPCC.2012.181.

12. A. M. Abu-Mahfouz, T. O. Olwal, A. M. Kurien, J. L. Munda and K. Djouani, "Toward developing a distributed autonomous energy management system (DAEMS)," AFRICON 2015, Addis Ababa, 2015, pp. 1-6, doi: 10.1109/AFRCON.2015.7332008.

13. T. Koga, K. Toyoda and I. Sasase, "Adaptive relay selection with energy and channel information in Energy Harvesting WSNs," 2015 12th Annual IEEE Consumer Communications and Networking Conference (CCNC), Las Vegas, NV, 2015, pp. 696-701, doi: 10.1109/CCNC.2015.7158063.

14. B. S. Ahir, R. Parmar and B. Kadhiwala, "Energy efficient clustering algorithm for data aggregation in wireless sensor network," 2015 International Conference on Green Computing and Internet of Things (ICGCIoT), Noida, 2015, pp. 683-688, doi: 10.1109/ICGCIoT.2015.7380550.

15. S. Sasaki, Y. Miyaji and H. Uehara, "Formulation of Energy Consumption in WSNs with Progress-Based Nearest Forwarding Policy," 2016 Fourth International Symposium on Computing and Networking (CANDAR), Hiroshima, 2016, pp. 161-167, doi: 10.1109/CANDAR.2016.0038.

16. Y. Zhu, E. Li and K. Chi, "Encoding Scheme to Reduce Energy Consumption of Delivering Data in Radio Frequency Powered Battery-Free Wireless Sensor Networks," in IEEE Transactions on Vehicular Technology, vol. 67, no. 4, pp. 3085-3097, April 2018, doi: 10.1109/TVT.2017.2776170.

17. S. K. Singh, P. Kumar, J. P. Singh and M. A. A. Alryalat, "An energy efficient routing using multi-hop intra clustering technique in WSNs," TENCON 2017 - 2017 IEEE Region 10 Conference, Penang, 2017, pp. 381-386, doi: 10.1109/TENCON.2017.8227894.

18. L. Cheklat, M. Amad, M. Omar and A. Boukerram, "Energy Efficient Physical Proximity based Chord Protocol for Data Delivery in WSNs," 2018 International Conference on Applied Smart Systems (ICASS), Medea, Algeria, 2018, pp. 1-6, doi: 10.1109/ICASS.2018.8652015.

19. A. Panchal, L. Singh and R. K. Singh, "RCH-LEACH: Residual Energy based Cluster Head Selection in LEACH for Wireless Sensor Networks," 2020 International Conference on Electrical and Electronics Engineering (ICE3), Gorakhpur, India, 2020, pp. 322-325, doi: 10.1109/ICE348803.2020.9122962. 